\title{
Singular density of states of disordered Dirac fermions in chiral models
}

\author{
Shinsei Ryu ${ }^{1}$ and Yasuhiro Hatsugai ${ }^{1,2}$ \\ ${ }^{1}$ Department of Applied Physics, University of Tokyo, 7-3-1 Hongo Bunkyo-ku, Tokyo 113-8656, Japan \\ ${ }^{2}$ PRESTO, JST, Saitama 332-0012, Japan \\ (Received 24 July 2001; published 4 December 2001)
}

\begin{abstract}
The Dirac fermion in the random chiral models is studied, which includes the random gauge field model and the random hopping model. We focus on a connection between continuum and lattice models to give a clear perspective for the random chiral models. Two distinct structures of density of states around zero energy, one is a power-law dependence on energy in the intermediate energy range and the other is a peak at zero energy, are revealed by an extensive numerical study for large systems up to $250 \times 250$. For the random hopping model, the above findings reconcile previous inconsistencies between the lattice and the continuum models.
\end{abstract}

DOI: 10.1103/PhysRevB.65.033301

PACS number(s): 72.15.Rn, 05.30.Fk, 71.23.-k

Dimensionalities and symmetries play central roles for universalities in the Anderson localization problem. More than two decades ago, Abrahams, Anderson, Licciardello, and Ramakrishnan ${ }^{1}$ presented the well-known scaling theory, predicting that electron wave functions always localize in one and two dimensions and metal-insulator transition occurs in three dimensions. However, even in two dimensions, delocalized states are also marginally allowed to appear when systems possess some special symmetries. For symmetries, it is often convenient to borrow terminologies from the random matrix theory which was first introduced by Wigner and Dyson. Recently, Altland and Zirnbauer ${ }^{2}$ have reported seven new symmetry classes in connection with a mathematical classification scheme of the Riemannian symmetric spaces.

Amongst these new symmetry classes, chiral models have attracted much attention as a novel exception for the scaling theory. Compared to the conventional models where randomness enters as an on-site potential, it resides on links (i.e., as a gauge field) in chiral models. This type of randomness may play important roles for the composite fermion theory of fractional quantum Hall effects and vortex states of dirty superconductors. In view of the localization problem, models with this randomness have a special symmetry, referred as chiral symmetry, and thereby belong to a new universality class. ${ }^{2}$ This symmetry is expected to affect localization properties of the systems drastically.

Several chiral models on a lattice, which are convenient for numerics, have been studied and interesting physics around zero-energy has been revealed. The density of states (DOS) of these models shows singularities at zero energy and the corresponding wave functions exhibit a delocalized multifractal behavior. Examples of these include the Gade's model ${ }^{3}$ the random flux model, ${ }^{4}$ and the $\pi$-flux model with link disorders. ${ }^{5}$ These models, defined on a two-dimensional square lattice, have the chiral symmetry which is conveniently stated as $\{H, \gamma\}=0$, where $\gamma$ is a matrix which changes the sign of wave functions on one of the sublattices. Consequently, for any realization of disorder, the energy spectrum is invariant under the transformation $E \rightarrow-E$. Therefore, given an eigenstate $\psi$ with energy $E, \gamma \psi$ is also an eigenstate with energy $-E$. This symmetry is responsible for the existence of delocalized states at zero energy.
Chiral models in a continuum space have also been investigated extensively. Especially, models that include the Dirac fermions have attracted interest. The Dirac fermion is a quasiparticle that appears in several interesting situations in condensed matter physics such as $d$-wave superconductivity, graphite sheets, gap-closing transitions in the quantum Hall effect, ${ }^{6}$ the Chalker-Coddington network model, ${ }^{7}$ and the mean field theory of the $t-J$ model. ${ }^{8}$ Effects of randomness are also of fundamental interest in these contexts. Since many analytic approaches such as a field theoretic one are applicable for the Dirac fermion, several interesting results have been obtained so far. Among them, there exist simple models that allow us to construct an explicit zero-mode wave function for any realization of disorder. ${ }^{9}$ Due to this advantage, it has been revealed that the zero-mode wave functions are not localized and exhibit a multifractal behavior. Moreover, there exists a transition in the multifractal spectrum ${ }^{10}$ and the density-density correlation ${ }^{11}$ as the disorder strength varied.

Although the delocalized multifractal nature of the exact zero energy states has been well established now, our knowledge for finite energy states, especially for DOS around zeroenergy, is still in confusion. For example, a continuum model where the Dirac fermions feel random gauge fields was studied in Refs. 9 and 12, and it was found that DOS exhibits a power-law dependence on energy. On the other hand, a similar model with species doubling was studied in Ref. 13 and a diverging DOS was found. From the lattice point of view, the corresponding model including Dirac fermions has not been studied so far. However, the random flux model, a lattice model where flux is randomly distributed for each plaquette, shows diverging DOS. ${ }^{14}$ A clear link between them is missing.

Another example is the case where the Dirac fermion is subject to imaginary vector potentials. For this case, there even exist inconsistent results between lattice and continuum models. Field theoretic studies ${ }^{13,15}$ predict diverging DOS at zero energy for any randomness strength. However, a numerical result for the random $\pi$-flux model, where a random hopping amplitude act as an imaginary vector potential, shows that the DOS behaves as a power law with its exponent dependent on the disorder strength for weak randomness. ${ }^{5}$ 
In this paper, we make an attempt to clarify the relationship between lattice models and continuum Dirac fermions, reconcile some inconsistent results in the previous studies, and thereby give a whole perspective for the localization problem of the two-dimensional (2D) chiral models. For these purposes, we consider lattice models that recover the random Dirac fermions in the continuum limit. We focus on DOS, especially around zero energy where quantum interference plays an important role and fully quantum mechanical treatments are necessary. We use the transfer-matrix method developed in Ref. 16. It allows us to handle large enough systems up to $250 \times 250$, which is indispensable to give reliable results for localization problems.

We realize Dirac fermions on a $2 \mathrm{D}$ square lattice via the $\pi$-flux model

$$
\mathcal{H}_{\text {pure }}=\sum_{\langle i j\rangle} c_{i}^{\dagger} t_{i j}^{\text {pure }} c_{j}+\text { H.c. }
$$

where $t_{j+\hat{x}, j}^{\text {pure }}=(-1)^{j_{x}}, \quad t_{j+\hat{y}, j}^{\text {pure }}=1, \quad$ and flux piercing a plaquette is $\pi$. The energy spectrum is given by $E= \pm 2 \sqrt{\cos ^{2} k_{x} a+\cos ^{2} k_{y} a}$, where k belongs to the magnetic Brillouin zone $(-\pi / a, \pi / a] \times(0, \pi / a]$ and $a$ is a lattice constant. In the continuum limit $a \rightarrow 0$, this includes the doubled massless Dirac fermions around $\mathbf{k}=(\pi / 2 a, \pi / 2 a)$ and $(-\pi / 2 a, \pi / 2 a)$. This realization is a minimum model for our purposes.

In the following, we will consider two types of disorder which live on a link: the random gauge field and the random hopping. We implement the random gauge field as

$$
t_{i j}^{r g}=t_{i j}^{\text {pure }} \exp \left[i a A_{i j}\right] .
$$

Taking a Coulomb gauge, we determine the random gauge field $A_{i j}$ via a scalar potential $\Phi$ on a dual lattice as $A_{j+\hat{x}, j}=\left(\Phi_{j+(\hat{x}+\hat{y}) / 2}-\Phi_{j+(\hat{x}-\hat{y}) / 2}\right) / a, A_{j+\hat{y}, j}=-\left(\Phi_{j+(\hat{x}+\hat{y}) / 2}\right.$ $\left.-\Phi_{j-(\hat{x}-\hat{y}) / 2}\right) / a$, where $\Phi$ is randomly chosen from a simple Gaussian distribution $P[\Phi] \propto \exp \left[-\left(a^{2} / 2 g\right) \Sigma_{<i j>}\left(\Phi_{i}\right.\right.$ $\left.\left.-\Phi_{j}\right)^{2} / a^{2}\right]$. In the continuum limit, this reduces to $P[\Phi]$ $\propto \exp \left[-(1 / 2 g) \int d^{2} x(\nabla \Phi)^{2}\right]$, a natural choice for effective field theoretic treatments. As stated above, this Hamiltonian possesses the chiral symmetry $\{\mathcal{H}, \gamma\}=0$ due to the special nature of randomness. Note also that time-reversal invariance is broken by the random magnetic flux.

In the continuum limit, the Hamiltonian is expressed as 5,13

$$
\mathcal{H}_{\text {cont }}=\int d^{2} x \Psi^{\dagger}\left(\begin{array}{cc}
0 & D \\
D^{\dagger} & 0
\end{array}\right) \Psi,
$$

where $D:=-i \partial_{x} \sigma_{x}-i \partial_{y} \sigma_{y}+A_{x}(\mathbf{x}) \sigma_{x}+A_{y}(\mathbf{x}) \sigma_{y}+M(\mathbf{x}) \sigma_{z}$ $+V(\mathbf{x}) \mathbf{1}, \sigma_{i=x, y, z}$ are the $2 \times 2$ Pauli matrices, and $\Psi$ is a four-component spinor. The coefficients $A_{x}, A_{y}, M$, and $V$ are arbitrary complex fields. This is the most general chiral symmetric form of a Dirac Hamiltonian in a continuum space. The chiral symmetry is, in the present basis, expressed as $\left\{\mathcal{H}, \sigma_{z} \otimes \mathbf{1}\right\}=0$. For the random gauge field model, $\left(A_{x}, A_{y}\right)$ is real and serves as the random gauge field. The other coefficients, $M$ and $V$, do not appear at the first order in the lattice constant $a$, but they are nonzero for the lattice models in general.
For strong enough randomness, this model is naturally related to another lattice model, the random flux model. (By the random flux model, we mean the model discussed in Refs. 4 and 14 which does not include Dirac fermions, while we simply call the model with Dirac fermions defined above as the random gauge field model.) To see this, note that the spatial correlation of flux per plaquette $\phi_{i}:=a^{2} \Delta_{i} \Phi_{i}$ is expressed as $\left\langle\phi_{i} \phi_{j}\right\rangle=a^{4} \Delta_{i} \Delta_{j} G(|i-j|)$, where $G(|i-j|):=\left\langle\Phi_{i} \Phi_{j}\right\rangle$ is a lattice Green's function for $\Phi$ and $\Delta_{i}$ is lattice Laplacian at a site $i$. Taking a continuum limit $a \rightarrow 0$, we find $\left\langle\phi_{i} \phi_{j}\right\rangle=0$ for finite $a|i-j|$, i.e., the spatial correlation of flux is short ranged. Moreover, for strong enough randomness, lattice fermions do not "remember" the original flux $\pi$ anymore, and we expect this model to exhibit a similar behavior to the random flux model where the flux for each plaquette is an independent random variable.

An effective sigma model via supersymmetry (SUSY) technique was studied in Ref. 13, predicting diverging DOS for a whole range of randomness strength. A similar model without species doubling $\mathcal{H}=\int d^{2} x \Psi^{\dagger}[\boldsymbol{\sigma} \cdot \mathbf{p}+\boldsymbol{\sigma} \cdot \mathbf{A}] \Psi$ has also been studied ${ }^{9,12}$ and power-law DOS with a disorderdependent exponent was proposed. On the other hand, the random flux model, which is expected to be the strong randomness limit of the present case, shows diverging DOS. ${ }^{14}$

We also consider the case where fermions on a $2 \mathrm{D}$ square lattice with $\pi$ flux per plaquette feel random hopping amplitudes

$$
t_{i j}^{r h}=t_{i j}^{\text {pure }}+\delta t_{i j}
$$

where $\delta t_{i j}$ is a real random variable. As for a probability distribution of $\delta t$, we consider a Gaussian distribution $P[\delta t] \propto \exp \left[-(\delta t)^{2} / 2 g^{2}\right]$.

In this case, $\left(A_{x}, A_{y}\right)$ in Eq. (2) is purely imaginary, and Dirac fermions are subject to an imaginary random gauge potential. Note that the total four-component Hamiltonian is Hermitian. Unlike the random gauge field model, timereversal symmetry is not broken for this model since we can take all matrix elements to be real in the real space.

As is the case of the random gauge field model, this model is connected to another lattice model for sufficiently strong randomness. For strong enough randomness, again, one expects that fermions no longer "remember" the original flux and show a similar behavior to that of the random hopping model without Dirac fermions.

Fukui $^{15}$ studied the corresponding continuum model by a replica nonlinear $\sigma$ model with a large number of fermion flavors and proposed that DOS at zero energy diverges and delocalized states exist at the band center for any randomness strength. A one-loop renormalization group (RG) study with SUSY method was also applied for this model in Ref. 13, predicting diverging DOS at zero energy as $\rho(E)$ $\sim\left(E_{R} / E\right) e^{-c \sqrt{\ln \left(E_{R} / E\right)}}$, where $E_{R}$ is a constant and $c$ depends on randomness. A conjectural RG flow beyond one-loop order was presented in Ref. 17 and similar results were obtained. The random hopping model without Dirac fermions, which we expect to be the strong randomness limit of the present model, also shows diverging DOS. ${ }^{3}$ On the other 


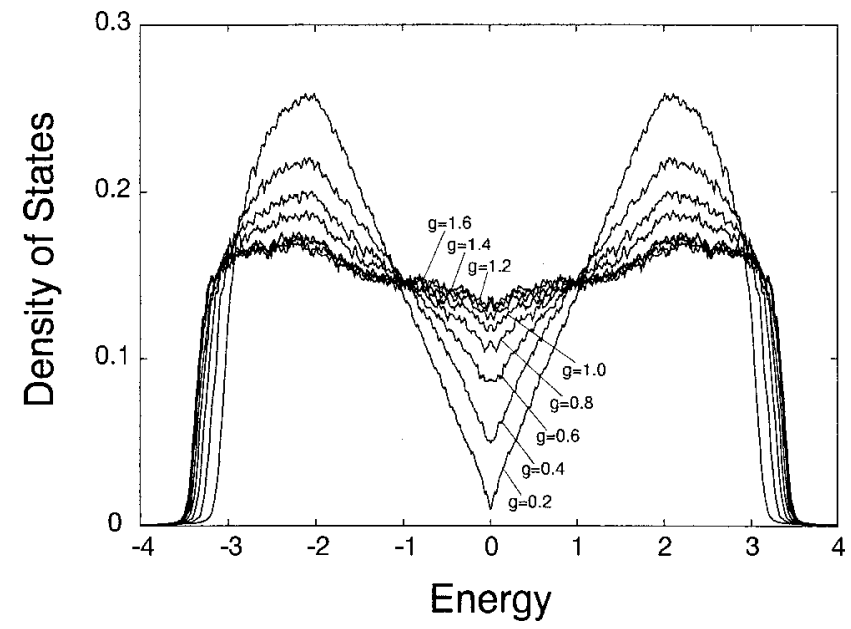

FIG. 1. Density of states for the $\pi$-flux model with a random gauge field on a $50 \times 50$ lattice for $g=0.2-1.6$ (from bottom to top at $E=0)$. The small imaginary part of energy $\delta$ is 0.01 . Quenched averaging is taken over 30 samples.

hand, according to a numerical study for the corresponding lattice model, vanishing DOS $\rho(E) \sim|E|^{\alpha}$ was observed with a disorder-dependent exponent $\alpha>0$ for weak randomness. ${ }^{5}$ We reexamine this problem by the transfer-matrix method.

A quantity of interest we investigate numerically is the random averaged DOS: $\langle\rho(E)\rangle:=\left\langle\left(1 / L^{2}\right) \Sigma_{i} \delta\left(E_{i}-E\right)\right\rangle$. For the pure $\pi$-flux model, the DOS vanishes linearly around zero energy due to the relativistic dispersion of Dirac fermions. Since for strong enough disorder, the present models are expected to exhibit similar properties to the random flux model or the random hopping model without Dirac fermions, both of which exhibit divergent DOS at zero energy, ${ }^{3,14}$ a natural question is how the vanishing DOS at zero energy becomes divergent as we increase the disorder strength $g$. It should be contrasted to the cases without Dirac fermions where the DOS of pure systems are already divergent at zero energy due to the van Hove singularities of a cosine band $E=2\left(\cos k_{x} a+\cos k_{y} a\right)$.

First, we discuss the random gauge field model, i.e., the $\pi$-flux model with a random gauge field as in Eq. (1). In Figs. 1 and 2, we present numerically calculated DOS for several $g$. In the transfer matrix method, the Green's function for a given energy $E$ is calculated at $E+i \delta$. We chose $\delta$ $\sim 0.01-0.0005$, which gives us enough resolution of energy. We used a square geometry rather than a quasi onedimensional one since we are mainly interested in purely two-dimensional properties.

As shown, there exist two structures. For over the wide range of the energy scale, the global feature of DOS changes from V shaped to flat; the latter is characteristic of the random flux model as expected. In addition, if we look more precisely for the very small region around zero energy, there exists another structure, i.e., a peak for sufficiently large $g$. Note that since we used a lattice with an even number of sites and adopted a periodic boundary condition, there is no exact zero-energy state, i.e., the peak found here is not an artifact by the special choice of boundary conditions. This peak structure is expected to smoothly connect to the known

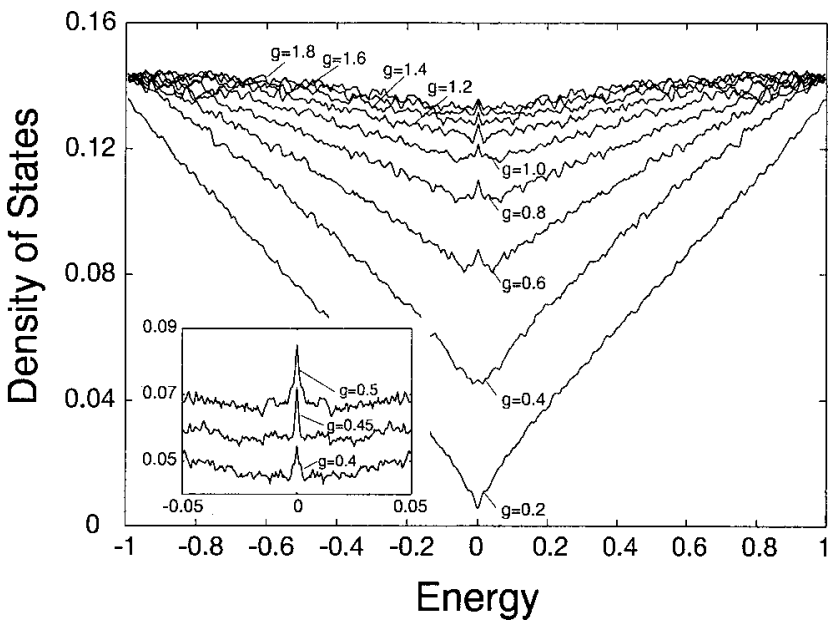

FIG. 2. Same as Fig. 1 on a $100 \times 100$ lattice for $g=0.1-1.8$, and $\delta=0.005$, averaged over 40 samples. Inset: same as Fig. 1 on a $250 \times 250$ lattice for $g=0.4,0.45,0.5$, and $\delta=0.0005$, averaged over 50 samples.

diverging DOS for the random flux model. The numerical results found here are consistent with the $\sigma$ model study via the SUSY technique in Ref. 13, where the divergence of DOS is predicted.

Now let us go on to the $\pi$-flux model with random hopping amplitudes [Eq. (3)]. Calculated DOS for systems up to $250 \times 250$ are shown in Figs. 3 and 4. Again, one recognizes two different structures as in the random gauge field model. Away from zero energy, the DOS exhibits a power-law behavior with a disorder-dependent exponent, as in Ref. 5. The whole DOS profile becomes divergent as the disorder strength increased, which is natural since the present model is expected to show similar behavior to that of Gade's model. On the other hand, as is the case with the random gauge field model studied above, within a very narrow range near zero energy, there exists another structure. As the disorder strength increased, a sharp peak at zero energy developed

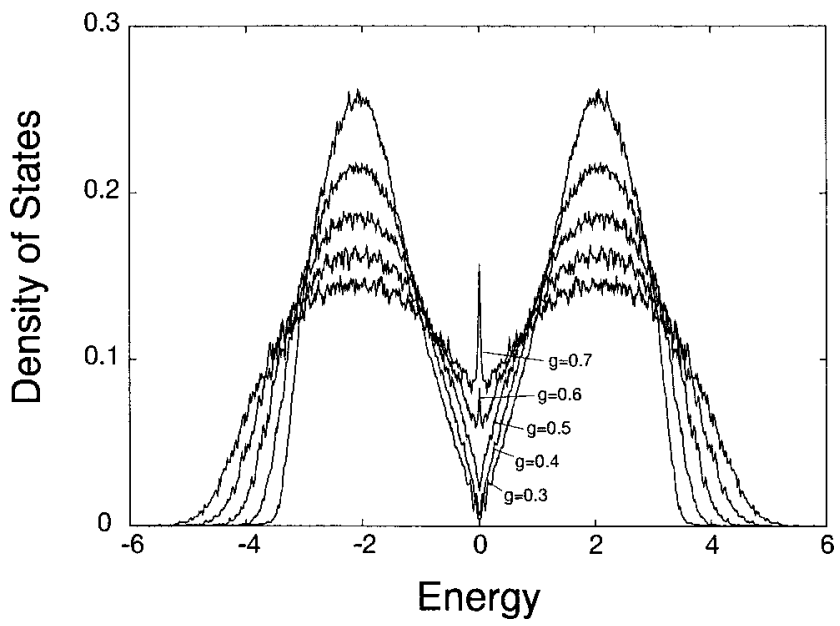

FIG. 3. Density of states for the random hopping model on a $50 \times 50$ lattice for $g=0.3-0.7$ (from bottom to top at $E=0$ ). The small imaginary part of energy $\delta$ is 0.01 . Quenched averaging is taken over 30 samples. 


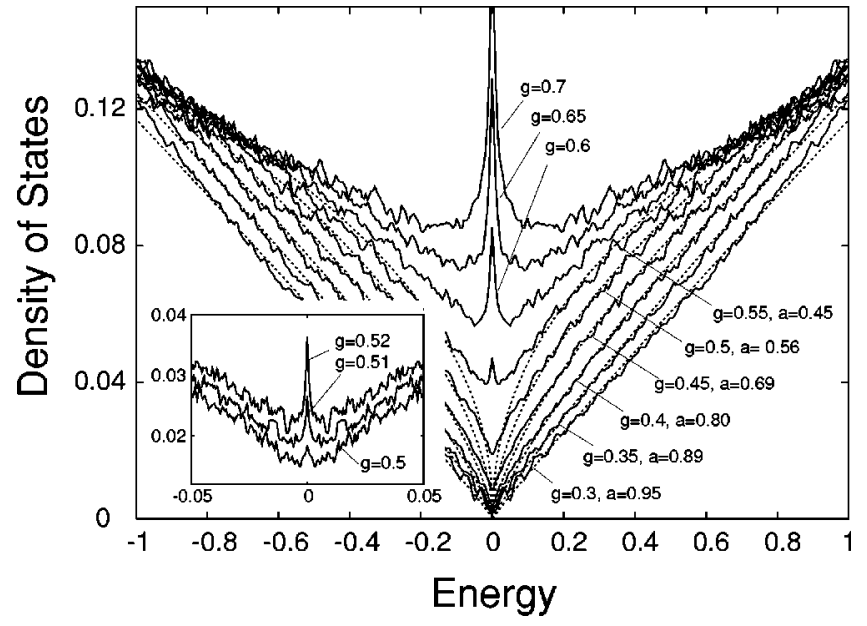

FIG. 4. Same as Fig. 3 on a $100 \times 100$ lattice for $g=0.3-0.7$, and $\delta=0.005$, averaged over 50 samples. Away from zero energy $(E>0.1)$ and for sufficiently weak randomness, data are fitted to a power law $\rho(E) \propto|E|^{a}$. Inset: same as Fig. 3 on a $250 \times 250$ lattice for $g=0.5,0.51,0.52$, and $\delta=0.0005$, averaged over 70 samples.

when $g$ reached about the order of the bandwidth. This behavior is independent of the global power-law profile away from the zero energy and before it turns divergent. These findings are consistent with the field theoretic analyses, especially with the SUSY approach where the divergence of the DOS is predicted and a power-law behavior appears in the intermediate region of the RG flow. We also investigated the case where $\delta t$ is uniformly distributed in $[-w / 2, w / 2]$. The results for this case are qualitatively similar to a Gaussian distribution.

We should compare the present case to dirty $d$-wave superconductors, which also include Dirac fermions. However, the underlying symmetry class is different in the random matrix theory. ${ }^{2}$ For dirty $d$-wave superconductors, all quasiparticle states are found to be localized, and a small energy scale around zero energy appears where quantum interference effects produce several critical DOS profiles depending on the details of randomness. ${ }^{18}$ This energy scale is determined by the diffusion constant in the diffusive regime and the localization length of quasiparticles. ${ }^{19}$ For the present case, on the other hand, zero-energy states are delocalized due to the chiral symmetry, and quantum interference effects give rise to diverging DOS at zero energy. The emergence of a power-law behavior away from zero energy is, from the effective field theory point of view, well described by the intermediate regime of the RG flow. ${ }^{13}$

In conclusion, we have investigated the DOS of the chiral models where the chiral symmetry plays crucial roles for the existence of the delocalized states at zero energy. We considered the lattice counterparts of continuum models including the Dirac fermions, the random gauge field model and the random hopping model, which have been well studied by field theoretic methods. Large-scale calculations by the transfer matrix method up to $250 \times 250$ lattices revealed the existence of the two distinct structures in the DOS: one is a power-law behavior in the intermediate energy range and the other is a peak at zero energy. Quantum interference plays crucial roles for these fine structures, which naive semiclassical treatments may miss. Our finding of the singular DOS at zero energy for the random hopping model reconciles inconsistencies between lattice and continuum models and thus establishes a clear connection between them.

We thank Y. Morita for fruitful discussions. The computation in this work has been partly done at the YITP Computing Facility and at the Supercomputing Center, ISSP, University of Tokyo.
${ }^{1}$ E. Abrahams, P. W. Anderson, D. C. Licciardello, and T. V. Ramakrishnan, Phys. Rev. Lett. 42, 673 (1979).

${ }^{2}$ A. Altland and M. R. Zirnbauer, Phys. Rev. B 55, 1142 (1997); M. R. Zirnbauer, J. Math. Phys. 37, 4786 (1996).

${ }^{3}$ R. Gade, Nucl. Phys. B 398, 499 (1993).

${ }^{4}$ T. Sugiyama and N. Nagaosa, Phys. Rev. Lett. 70, 1980 (1993); Y. Avishai, Y. Hatsugai, and M. Kohmoto, Phys. Rev. B 47, 9561 (1993).

${ }^{5}$ Y. Hatsugai, X.-G. Wen, and M. Kohmoto, Phys. Rev. B 56, 1061 (1997); Y. Morita and Y. Hatsugai, Phys. Rev. Lett. 79, 3728 (1997).

${ }^{6}$ Y. Hatsugai and M. Kohmoto, Phys. Rev. B 42, 8282 (1990).

${ }^{7}$ C.-M. Ho and J. T. Chalker, Phys. Rev. B 54, 8708 (1996).

${ }^{8}$ I. Affleck and J. B. Marston, Phys. Rev. B 37, 3774 (1988).

${ }^{9}$ A. W. W. Ludwig, M. P. A. Fisher, R. Shankar, and G. Grinstein, Phys. Rev. B 50, 7526 (1994).

${ }^{10}$ C. Chamon, C. Mudry, and X.-G. Wen, Phys. Rev. Lett. 77, 4194 (1996); H. E. Castillo, C. Chamon, E. Fradkin, P. M. Goldbart, and C. Mudry, Phys. Rev. B 56, 10668 (1997).
${ }^{11}$ S. Ryu and Y. Hatsugai, Phys. Rev. B 63, 233307 (2001).

${ }^{12}$ C. Mudry, C. Chamon, and X.-G. Wen, Nucl. Phys. B 466, 383 (1996).

${ }^{13}$ S. Guruswamy, A. LeClair, and A. W. W. Ludwig, Nucl. Phys. B 583, 475 (2000).

${ }^{14}$ A. Furusaki, Phys. Rev. Lett. 82, 604 (1999).

${ }^{15}$ T. Fukui, Nucl. Phys. B 562, 477 (1999).

${ }^{16}$ L. Schweitzer, B. Kramer, and A. MacKinnon, J. Phys. C 17, 4111 (1984).

${ }^{17}$ A. LeClair, cond-mat/0011413 (unpublished).

${ }^{18}$ W. A. Atkinson, P. J. Hirschfeld, A. H. MacDonald, and K. Ziegler, Phys. Rev. Lett. 85, 3926 (2000); A. G. Yashenkin, W. A. Atkinson, I. V. Gornyi, P. J. Hirschfeld, and D. V. Khveshchenko, ibid. 86, 5982 (2001); See also B. Huckestein and A. Altland, cond-mat/0007413 (unpublished), where a power-law behavior of the DOS in dirty $d$-wave superconductors is discussed for the intermediate energy regime.

${ }^{19}$ T. Senthil and M. P. A. Fisher, Phys. Rev. B 60, 6893 (1999). 